\title{
The efficacy of ginkgolide $B$ in the acute treatment of migraine aura: an open preliminary trial
}

\author{
Gianni Allais • Giovanni D'Andrea • \\ Maurizio Maggio $\cdot$ Chiara Benedetto
}

(C) Springer-Verlag Italia 2013

\begin{abstract}
In this open trial we evaluated the possible efficacy of Ginkgolide B in the treatment of acute aura in a group of patients suffering from migraine with aura, considering in particular the effect of the treatment on aura duration. Twenty-five patients (16 females, 9 males, mean age $39.7 \pm 13.5$ years, range 18-65) suffering from migraine with aura were enrolled in the study. The diagnosis was made according to the diagnostic criteria of the international classification of headache disorders, second edition (ICHD-II), for typical aura with migraine headache $(n=19)$ or typical aura without headache $(n=6)$. Patients were asked to use a diary card to register the exact duration of the aura symptoms in two consecutive attacks of aura. In the first one, they only took note of the duration of neurological symptoms in minutes. In the following attack, they were instructed to take orally, immediately at the onset of the first symptoms of aura, two capsules of a combination of $60 \mathrm{mg}$ Ginkgo biloba terpenes phytosome, $11 \mathrm{mg}$ coenzyme Q 10 and $8.7 \mathrm{mg}$ vitamin B2 (Migrasoll ${ }^{\circledR}$ ). Aura duration (expressed in minutes) was
\end{abstract}

G. Allais $(\square) \cdot$ C. Benedetto

Department of Gynecology and Obstetrics,

Women's Headache Center, University of Turin,

Via Ventimiglia 3, 10126 Turin, Italy

e-mail: gb.allais@tiscali.it

G. D'Andrea

Research and Innovation (R\&I), Padua, Italy

M. Maggio

Department of Neurology, Ivrea Hospital, Ivrea, Italy significantly $(p<0.001)$ reduced by Migrasoll ${ }^{\circledR}$ intake, being $33.6 \pm 11.5$ in the first untreated attack and $21.9 \pm 11.8$ during the second attack. In general, there was a marked amelioration of the features of the neurological symptoms of aura in the treated attack. In four patients $(18.1 \%)$ suffering from typical aura with migraine, the pain phase disappeared. Among the patients who completed the study no serious adverse events were reported.

Keywords Acute treatment - Ginkgolide B - Migraine with aura Platelet activating factor
Abbreviations
CNS Central nervous system
MwA Migraine with aura
PAF Platelet activating factor
SD Spreading depression

\section{Introduction}

Migraine with aura (MwA) is a primary headache disorder affecting up to $30 \%$ of migraine sufferers [1]. The International Headache Society's (IHS) diagnostic criteria for MwA [2] provide a clinical description of the aura, with the disorder's most distinctive features being transient, unilateral or bilateral visual, sensory or motor symptoms due to a possible recurrent, reversible, idiopathic dysfunction of the cortex or brainstem. Usually, prophylactic treatment is not necessary in a great percentage of patients because the frequency of the attacks is low, i.e. 3-4 attacks/year. When the frequency of the MwA increases, lamotrigine, an antiepileptic drug, is effective in reducing the number of auras [3]. A specific treatment capable of significantly 
reducing the length of the auras remains an unsolved problem. This treatment is necessary because in many patients the abrupt occurrence of visual or somatic symptoms of the aura causes severe anxiety and distress (sudden loss of vision in the visual field, strength in one arm, etc). This occurs more often when the aura lasts $1 \mathrm{~h}$ or even more (at times the symptoms of aura are prolonged and may last days). In addition, although infrequently, aura may trigger a cerebral ischemic attack with permanent neurological deficits (migraine stroke) [3].

Functional evidence suggests that the visual aura is a neuronal event similar to the spreading depression (SD) triggered on the cortex of the rabbit by potassium described by Leao [4]. The initial event is a spontaneous neuronal depolarization moving slowly $(3 \mathrm{~mm} / \mathrm{min}$ ) on the occipital cortex, which has the clinical counterpart in the scintillating contour (positive scotoma). Negative scotoma (the dark area inside scintillations) may be the subsequent suppression of neuronal function [5]. Neurophysiological studies, using neuromagnetometer and transcranial stimulation of the occipital cortex, support the hypothesis that a neuronal hyperexcitability [6] is the predisposing factor that causes the initial cortical event [7]. Glutamic and aspartic acids are excitatory amino acids that elicit and support SD in animal models. Elevated levels of these excitatory neurotransmitters have been found in platelets of patients suffering from MwA [8]. Platelets are a fairly good model for studying the glutamate function of the neuron. Thus, if these platelet findings mirror the similar abnormal levels in neurons, it is possible that an increased release of glutamate from dendrites or soma depolarizes occipital cortex mediating SD.

The predisposing factors for MwA attacks are unknown. However, increased susceptibility to platelet dense bodies (in which glutamate is contained) release [9], together with a low threshold of platelet to aggregating agents as platelet activating factor (PAF) and ADP, may predispose to auras MwA sufferers [10].

Ginkgolide B, an herbal constituent extract from Ginkgo biloba tree leaves, is a natural modulator of the action of glutamate in the CNS [11]. Moreover, it is a powerful antiplatelet activating factor (PAF). PAF is a potent proinflammatory and nociceptive agent released during the inflammation process [11]. Indeed, PAF and glutamate, released from platelets and leukocytes in the blood and from the glia in the CNS, may play a role in the occurrence and propagation of SD in the cortex. In addition, one study has shown that Ginkgolide B is useful in the prophylaxis of MwA [12]. On the basis of this evidence, in an open trial we evaluated the possible efficacy of Ginkgolide B in the treatment of acute aura in a group of patients suffering from MwA, considering in particular the effect of the treatment on aura duration.

\section{Patients and methods}

Twenty-five patients (16 females, 9 males, mean age $39.7 \pm 13.5$ years, range 18-65) suffering from migraine with aura were enrolled in the study. The diagnosis was made according to the diagnostic criteria of the international classification of headache disorders, second edition (ICHD-II) [2], for typical aura with migraine headache $(n=19)$ or typical aura without headache $(n=6)$.

Criteria for admission to the study were as follows: age ranging from 18 to 65 years; a minimum 2-year history of migraine with and/or aura without migraine; auras occurring at least four times a year; length of neurological symptoms of at least $15 \mathrm{~min}$ in the anamnesis; in patients suffering from typical aura with migraine, the aura must always precede the pain and not accompany it; no past or present diseases, and in particular no history of cerebral focal activation; no pregnancy or lactation. All patients gave their informed consent to participate in the study.

Patients were free of prophylactic drugs during the whole study.

They were asked to use a diary card to register the exact duration of the aura symptoms in two consecutive attacks of aura. In the first one, they only took note of the duration of neurological symptoms in minutes. In the following attack, they were instructed to take orally, immediately at the onset of the first symptoms of aura, two capsules of a combination of $60 \mathrm{mg}$ Ginkgo. biloba terpenes phytosome, $11 \mathrm{mg}$ coenzyme Q 10 and $8.7 \mathrm{mg}$ vitamin B2 (Migrasoll $\left.{ }^{\circledR}\right)$. No restriction was placed on analgesic intake during the pain phase. The statistical evaluation was performed using a $t$ test for repeated measures. All values given in the following text are reported as arithmetic means ( $\pm 1 \mathrm{SD})$.

\section{Results}

Twenty-five patients entered the study. Three of them withdrew from the trial: one because for more than 1 year he had never had auras, and two because they were lost to follow-up. The results shown in this paper are therefore related to the remaining 22 patients (14 females, 8 males, mean age $37.9 \pm 13.8)$.

Aura duration (expressed in minutes) was significantly $(p<0.001)$ reduced by Migrasoll ${ }^{\circledR}$ intake, being $33.6 \pm$ 11.5 in the first untreated attack and $21.9 \pm 11.8$ during the second attack.

Individual changes in MA symptomatology are not considered in this paper. Nevertheless, it is noteworthy that in general there was a marked amelioration of the features of the neurological symptoms of aura in the treated attack.

In four patients $(18.1 \%)$ suffering from typical aura with migraine, the pain phase disappeared. 
Among the patients who completed the study no serious adverse events were reported.

\section{Discussion}

This study showed that administering two capsules of Migrasoll $^{\circledR}$ containing $120 \mathrm{mg}$ of the terpenic fraction (Ginkgolide B) of the Ginkgo biloba tree to patients presenting an acute aura significantly reduced the length of their neurological symptoms in more than $60 \%$ of the cases. In $18 \%$ of patients suffering from typical aura followed by migraine the pain phase totally disappeared.

The reason why Ginkgolide B is able to relieve the aura symptoms so quickly is an intriguing matter. One explanation is that Ginkgolide $\mathrm{B}$ along with other terpene trilactones such as bilobalide, A, C, and flavonoids, all constituents of Ginkgo biloba leaves possess specific pharmacological actions on PAF matrix metalloproteinases (MMPs), and glutamate activity on NMDA receptors [13, 14], all of which seem to be implicated in the pathogenesis of aura. In terms of pharmacological properties, Ginkgolide $\mathrm{B}$ is reported to be the most potent constituent of Ginkgo biloba and is maximally concentrated in the terpene fraction.

One important limitation of this study is that we do not have data about the plasma concentration of Ginkgolide B at the various times after our patients ingested the capsules.

While we cannot rule out that our good results are due to a placebo effect, this may not be the case since a previous, controlled multicenter study utilizing sumatriptan subcutaneous injection specifically to treat the aura did not show the placebo effect. In fact, sumatriptan proved ineffective in reducing the length of the aura symptoms [15].

In conclusion, for the first time, this small open study suggests that the acute attack of aura can be successfully treated with an oral administration of Ginkgolide B. Nevertheless, these results should be interpreted with caution and confirmed with a placebo-controlled trial involving a higher number of patients.

Conflict of interest G. D'Andrea is the Medical Director of Pharmaval Srl.

\section{References}

1. Launer LJ, Terwindt GM, Ferrari MD (1999) The prevalence and characteristics of migraine in a population based cohort: the GEM study. Neurology 53:537-542

2. Headache Classification Subcommittee of the International Headache Society (2004) The international classification of headache disorders, 2nd edn. Cephalalgia 24(Suppl 1):9-169

3. D'Andrea G, Bonavita V, Rigamonti A, Bussone G (2003) Treatment of migraine with aura comments and prospectives. Neurol Sci 23:271-278

4. Leao AAP (1944) Spreading depression of activity of cerebral cortex. J Neurophysiol 7:279-390

5. Olesen J, Friberg L, Olesen TS et al (1990) Timing and topography of cerebral blood flow, aura, and headache during migraine attacks. Ann Neurol 28:791-798

6. Welch KMA, D'Andrea G, Terpley N, Barkley G, Ramadan NM (1990) The concept of migraine as a state of central neuronal hyperexcitability. Neurol Clin 8:817-828

7. Aurora SK, Ahmad BK, Welch KMA, Bhardhwaj P, Ramadan NM (1998) Trancranial magnetic stimulation confirms hyperexcitability of occipital cortex in migraine. Neurology 50: $1111-1114$

8. Cananzi AR, D'Andrea G, Perini F, Zamberlan F, Welch KMA (1995) Plasma and platelet levels of glutamate and glutamine in migraine with and with out aura. Cephalagia 15:132-135

9. D'Andrea G, Perini F, Terrazzino S, Nordera GP (2004) Contributions of biochemistry to the pathogenesis of primary headaches. Neurol Sci 25:S89-S92

10. D'Andrea G, Hasselmark L, Alecci A, Cananzi A, Perini F, Welch KMA (1994) Platelet secretion from dense and $\alpha$-granules in vitro in migraine with and without aura. J Neurol Neurosurg Psych 57:557-561

11. Xia S-H, Fang D-C (2007) Pharmacological action and mechanism of ginkgolide B. Clin Med J 120(10):922-928

12. D'Andrea G, Bussone G, Allais G, Aguggia M, d'Onofrio F et al (2009) Efficacy of ginkgolide b in the prophylaxis of migraine with aura. Neurol Sci 30(Suppl 1):S121-S124

13. Cho H-J, Nam K-S (2007) Inhibitory effect of ginkgolide B on platelet aggregation and cGMP-dependent manner by activated MMp-9. J Biochem and Molecular Biol 40:678-683

14. Xiao ZY, Dom CK, Xiao XW, LinYZ et al (2006) Effetti estratto di Ginkgo biloba contro tossicità indotta da recettori NMDA e loro meccanismo Zhonghua Yi Xue Za Zhi. 19; 86:247924-84

15. Bates D, Ashford E, Dawson R, Ensink FB et al (1994) Subcutaneous sumatriptan during the migraine aura. Sumatriptan Aura Study Group. Neurology 44(9):1587-1592 